Archived version from NCDOCKS Institutional Repository http://libres.uncg.edu/ir/asu/

\title{
Appalachỉan
}

B O O N E, NORT H C A R O L I N A

\section{Eleven Diverse Nuclear-Encoded Phylogenetic Markers For The Subfamily Panicoideae (Poaceae)}

\author{
Authors \\ Matt C. Estep, Dilys M. Vela Diaz, Jinshun Zhong And Elizabeth A. Kellogg
}

\begin{abstract}
Premise of the study: Polyploidy is common in the grasses and low-copy nuclear loci are needed to further our understanding of phylogenetic relationships.
\end{abstract}

- Methods and Results: Genetic and genomic resources were combined to identify loci known to influence plant and inflorescence architecture. Degenerate primers were designed and tested to amplify regions of 11 nuclear-encoded loci across the panicoid grasses.

- Conclusions: The primers designed in this study amplify regions of a diverse set of genes within the panicoid grasses. Properly employed, these markers will allow the identification of allopolyploid taxa and their diploid progenitors.

Matt C. Estep, Dilys M. Vela Diaz, Jinshun Zhong And Elizabeth A. Kellogg. (2012) "Eleven Diverse Nuclear-Encoded Phylogenetic Markers For The Subfamily Panicoideae (Poaceae)" American Journal of Botany. Version of Record Available At www.amjbot.org [doi:10.3732/ajb.1200186] 
Subfamily Panicoideae Link (Poaceae) is a diverse group of mainly tropical grasses that includes $\sim 3300$ species, grouped into 200 genera (Grass Phylogeny Working Group, 2001). This subfamily of the grasses contains crop species (Zea mays, Sorghum bicolor, Setaria italica), biofuel stocks (Panicum virgatum and Saccharum sp.), agricultural weeds (Setaria viridis, Rottboellia cochinchinesis, and Sorghum halapense), and dominant species in tropical grasslands around the world (Andropogon sp., Hyparrhenia sp., and Schizachyrium sp.). Polyploidy and reticulate evolution are common in the grasses, requiring data from low-copy nuclear loci to resolve relationships. Although the internal transcribed spacer of the ribosomal genes (ITS) is widely used, its high copy number and concerted evolution make it inadequate for this purpose (Álvarez and Wendel, 2003).

Over the past decade, extensive genetic and genomic resources have been developed to study agriculturally important grasses. Genome sequences are available for several crop species in the panicoids, including maize (Zea mays), sorghum (Sorghum bicolor), and foxtail millet (Setaria italica). These genome sequences can be used as a reference for primer design using databases such as Phytozome and the National Center for Biotechnology Information (NCBI) databases. In addition to genome sequences, the genetics community has conducted extensive studies to identify genes that play functional roles in many agriculturally and evolutionarily important traits. Many of these loci influence plant and inflorescence architecture, the same traits used by botanists to define groups and species. Combining these resources allowed us to identify and design primers for 11 new single to low-copy nuclear loci for use within the panicoid grasses.

\section{METHODS AND RESULTS}

We identified genes from the literature that are known to influence grass morphology and inflorescence architecture, resulting in a large number of candidate loci. This list was reduced to $\sim 20$ gene candidates by removing genes that are part of large gene families. Published sequences (usually cDNA) from these studies were then used to query a database of translated nucleotide sequences from plant genome projects using Phytozome 8.0 (http://www.phytozome. net). This allowed the identification of homologous sequences in the three panicoid grass taxa for which genomes are available (Setaria, Sorghum, and Zea). Sequences were downloaded and used to query the high-throughput genomic sequences (HTGS) database on NCBI for the maize genome to aid in identifying exon/intron boundaries. All sequences were then translated and aligned using MUSCLE, as implemented in Geneious Pro 5.5.6 (BioMatters, Auckland, New Zealand). Alignments were then trimmed to exclude ambiguously aligned regions and to avoid large introns. Portions of coding sequence of $\sim 800-1200$ bp in the reference alignment were identified for primer design. The web-based software Primaclade was used to develop multiple primer pairs for each locus (Gadberry et al., 2005).

Each primer pair was then tested on a diverse group of 10 taxa (Appendix 1). PCR was carried out using primer-specific conditions in $25-\mu \mathrm{L}$ reactions containing $100 \mathrm{ng}(1 \mu \mathrm{L})$ of template DNA using GoTaq Flexi DNA polymerase kits (Promega Corporation, Madison, Wisconsin, USA). Reactions included $5 \times$ green flexi buffer $(5 \mu \mathrm{L}), 25 \mathrm{mM} \mathrm{MgCl} 2(2 \mu \mathrm{L}), 2.5 \mathrm{mM}$ dNTPs $(2 \mu \mathrm{L}), 10 \mu \mathrm{M}$ of each primer $(1 \mu \mathrm{L})$, and 1 unit of $\operatorname{Taq}(0.2 \mu \mathrm{L})$. Five molar betaine $(2.5 \mu \mathrm{L})$ and dimethyl sulfoxide (DMSO; $1.25 \mu \mathrm{L}$ ) were added to reactions when primer sequences contained a GC content above $50 \%$. Reactions were performed at an annealing temperature equal to the melting temperature $\left(T_{\mathrm{m}}\right)-5^{\circ} \mathrm{C}$ or using a touchdown protocol. Thermocycler conditions for standard reactions were: initial denature at $96^{\circ} \mathrm{C}$ for $5 \mathrm{~min}$, followed by 32 cycles of denature at $96^{\circ} \mathrm{C}$ for $1 \mathrm{~min}$, annealing at $T_{\mathrm{m}}-5^{\circ} \mathrm{C}$ for $1 \mathrm{~min}$, elongation at $72^{\circ} \mathrm{C}$ for $1 \mathrm{~min}$, and a final elongation step at $72^{\circ} \mathrm{C}$ for $5 \mathrm{~min}$. Thermocycler conditions for touchdown reactions were similar except three rounds of five cycles were used starting with an annealing temperature of $68^{\circ} \mathrm{C}$ and reducing it each round by $3^{\circ} \mathrm{C}$; these 15 cycles were then followed by 25 cycles at an annealing temperature of $58^{\circ} \mathrm{C}$. Twelve primer pairs produced a single band for all 10 taxa upon gel electrophoresis (Table 1). These bands were purified using a QIAGEN gel extraction kit, following the manufacturer's protocol (Valencia, California, USA). Purified products were cloned using pGEM-T Easy Vector and transformed into JM109 High-Efficiency Competent Cells, following manufacturer's protocols (Promega 
TABLE 1. Primer sequences and PCR conditions for 11 nuclear-encoded loci.

\begin{tabular}{|c|c|c|c|c|}
\hline Gene name & Abbreviation & Primer sequences $\left(5^{\prime}-3^{\prime}\right)$ & $\bar{T} \overline{T_{\mathrm{a}}\left({ }^{\circ} \mathrm{C}\right)}$ & GC rich \\
\hline \multirow[t]{2}{*}{ Aberrant Panicle Organization 1} & apo 1 & F: TCTACTCGTCGCCGTTCCT & 58 & Yes \\
\hline & & R: AGGAACCTCCTCATCGGC & & \\
\hline \multirow[t]{2}{*}{ Dwarf8 } & $d 8$ & F: ATGAAGCGCGAGTACCAAGAC & TD $68-55$ & Yes \\
\hline & & $\begin{array}{l}\text { R: GAAGTCGACGCGGATGGT } \\
\text { F2seq: CWTGCTGTCCGAGSTCAAC } \\
\text { R2seq: AGTTCTCCTGCTGCACGG }\end{array}$ & 58 & No \\
\hline \multirow[t]{4}{*}{ Erect Panicle } & ep2-ex7 & $\mathrm{F}:$ GAGCAGACTCCAAGTTCTGGT & 58 & No \\
\hline & & R: GGATGATTGCCTGCTCAAAT & & \\
\hline & ep2-ex8 & $\mathrm{F}:$ TCAATCGAGGAGGTCACAATC & 58 & No \\
\hline & & R: TGAGGCAGTGGAAGCAGATA & & \\
\hline \multirow[t]{2}{*}{ Floral Organ Regulator 1} & for 1 & $\mathrm{~F}:$ ACMTGCASGCGCTGCTGAG & 58 & Yes \\
\hline & & R: GTTGTGGGASAGGTCCAGGTA & & \\
\hline \multirow[t]{2}{*}{ Liguleless 1} & $\lg 1$ & F: ATCTSCTGGATGAGTTCGAYGA & 58 & No \\
\hline & & R: AAGTCGAGATCRAACATMGCCTG & & \\
\hline \multirow[t]{2}{*}{ Monoculm } & moc1 & $\mathrm{F}:$ CTGGCGTACAACCAGATCG & 58 & Yes \\
\hline & & R: GTCTGCCAGCCGAGGAAG & & \\
\hline \multirow[t]{2}{*}{ Ramosa 1} & $r a 1$ & $\mathrm{~F}:$ CGCCRCAGRTAAGGTCGTC & 52 & No \\
\hline & & R: GCCCAGTCTAAGCTGAAGATCC & & \\
\hline \multirow[t]{2}{*}{ Ramosa 1 Enhancer Locus2 } & rel2 & F: ATGTCKTCKCTKAGCAGGGA & 58 & Yes \\
\hline & & R: RAGCATGATATTCCGRGCT & & \\
\hline \multirow[t]{2}{*}{ Ramosa2 } & $r a 2$ & $\mathrm{~F}:$ ATGGCRTCSYCGTCGAGCACC & 57 & No \\
\hline & & R: CATGCTGCTGTCTCCYCCTTCC & & \\
\hline \multirow[t]{3}{*}{ Retarded Palea 1} & rep 1 & F1: САСТTCTTCYCCRGCCAYGSCC & TD $57-52$ & No \\
\hline & & F2: CAAYTCYGARACNCTGGAGGC & & \\
\hline & & R: GTCSARGAAATCRYCRYCKTC & & \\
\hline \multirow[t]{2}{*}{ Vanishing Tassel2 } & $v t 2$ & $\mathrm{~F}:$ TACATCGAGCTGGTGTGCTC & 58 & No \\
\hline & & R: AKATGTCGTAGGCRTCGTCC & & \\
\hline
\end{tabular}

Note: $T_{\mathrm{a}}=$ annealing temperature .

Corporation). Eight positive clones for each PCR product were sequenced in both directions using universal primers (M13, Sp6, or T7) on an ABI3730 DNA sequencer (Applied Biosystems, Carlsbad, California, USA). Chromatograms were trimmed of vector and resulting sequences were translated and aligned with the reference sequences using MUSCLE, as implemented in Geneious Pro 5.5.6 (BioMatters). The resulting sequences were compared using a maximum likelihood tree, and an exemplar was chosen for each distinct clade to represent a species or paralogues within a species. Regions not suitable for phylogenetic analysis (introns, ambiguously aligned regions, and primer annealing sites) were annotated on a consensus sequence and excluded during character analysis (Fig. 1). Known protein domains were also identified using Pfam databases and annotated on the consensus sequence (Punta et al., 2012). Statistics describing each alignment were generated using parsimony in PAUP* 4.0 (Swofford, 2002). An uncorrected distance matrix was calculated to identify the percent variation between different sequences (Table 2).

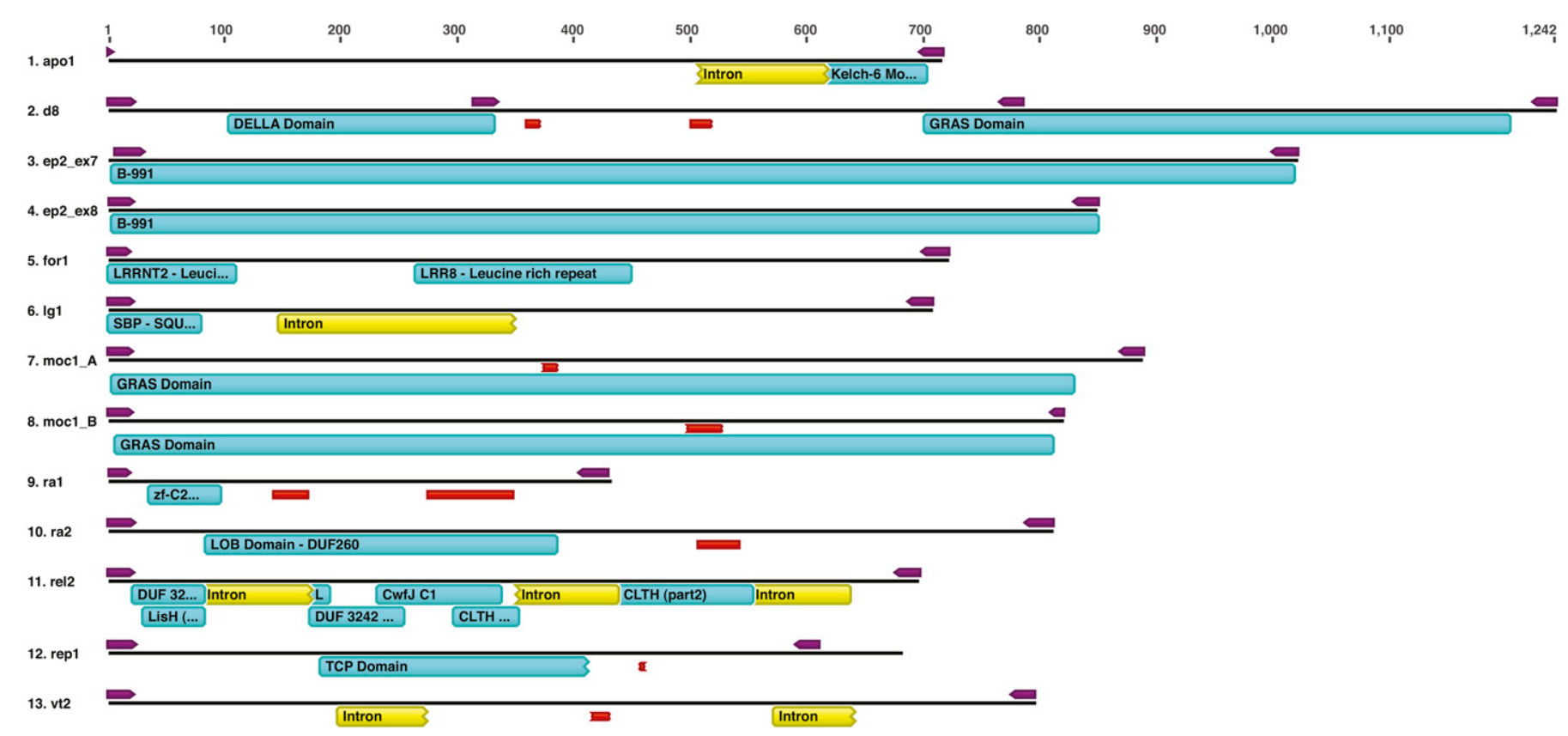

Fig. 1. Annotation of consensus sequences generated from 13 alignments. Sequence length (black line), primer binding locations (purple arrows), introns (yellow), ambiguously aligned regions (red), and Pfam domains (turquoise) are indicated. 
TABLE 2. Characteristics of 13 sequence alignments generated from 10 diverse taxa and five reference sequences for 11 nuclear-encoded genes.

\begin{tabular}{|c|c|c|c|c|c|c|c|c|c|}
\hline \multirow[b]{3}{*}{ Locus } & \multicolumn{3}{|c|}{ Alignment } & \multicolumn{4}{|c|}{ Characters } & & \\
\hline & \multirow[b]{2}{*}{ Length (bp) } & \multirow[b]{2}{*}{ Intron } & \multirow[b]{2}{*}{ Ambiguous region } & \multirow[b]{2}{*}{ Total excluded } & \multirow[b]{2}{*}{ Constant } & \multicolumn{2}{|c|}{ Parsimony variable } & \multicolumn{2}{|c|}{ Pairwise variation } \\
\hline & & & & & & Uninformative & Informative & Min & Max \\
\hline apo1 & 860 & 1 & 0 & 208 & 512 & 78 & 62 & 0.016 & 0.115 \\
\hline$d 8$ & 1273 & 0 & 2 & 39 & 918 & 190 & 126 & 0.031 & 0.101 \\
\hline ep2_ex7 & 1054 & 0 & 0 & 0 & 746 & 199 & 109 & 0.018 & 0.158 \\
\hline ep2_ex8 & 869 & 0 & 0 & 0 & 640 & 144 & 85 & 0.007 & 0.128 \\
\hline for 1 & 721 & 0 & 0 & 0 & 551 & 91 & 79 & 0.036 & 0.117 \\
\hline $\lg 1$ & 880 & 1 & 0 & 322 & 431 & 51 & 76 & 0.031 & 0.126 \\
\hline $\operatorname{moc} 1 \mathrm{~A}$ & 933 & 0 & 1 & 31 & 714 & 125 & 63 & 0.036 & 0.100 \\
\hline$m o c 1 \mathrm{~B}$ & 894 & 0 & 1 & 48 & 606 & 143 & 97 & 0.038 & 0.137 \\
\hline$r a 1$ & 505 & 0 & 2 & 129 & 234 & 90 & 52 & 0.030 & 0.281 \\
\hline$r a 2$ & 849 & 0 & 1 & 39 & 643 & 86 & 81 & 0.014 & 0.110 \\
\hline rel2 & 790 & 3 & 0 & 355 & 388 & 27 & 20 & 0.017 & 0.055 \\
\hline rep 1 & 864 & 0 & 1 & 48 & 527 & 145 & 144 & 0.055 & 0.209 \\
\hline$v t 2$ & 1101 & 2 & 1 & 450 & 510 & 60 & 81 & 0.034 & 0.103 \\
\hline
\end{tabular}

A total of 13 primer pairs was generated for amplifying (12 pairs) and sequencing (1 pair) regions of 11 distinct loci found in the panicoid grasses (Table 1). These loci include Aberrant panicle organization 1 (apo1), Dwarf8 (d8), two exons of Erect panicle2 (ep2), Floral organ regulator1 (for1), liguleless 1 (lg1), Monoculm1 (moc1), Ramosa1 (ra1), Ramosa1 Enhancer locus 2 (rel2), Ramosa2 (ra2), Retarded palea1 (rep1), and Vanishing Tassel2 ( vt2) (Moreno et al., 1997; Peng et al., 1999; Jang et al., 2003; Li et al., 2003; Ikeda et al., 2005; Vollbrecht et al., 2005; Bortiri et al., 2006; Yuan et al., 2009; Gallavotti et al., 2010; Zhu et al., 2010; Phillips et al., 2011). Sequences generated with these primers produced 13 alignments with a combined alignable length of almost 10000 bp. Two of these alignments (moc1 A \& B) are generated with a single pair of primers and represent a duplication that occurred prior to the divergence of grasses. The rep 1 primers occasionally amplify multiple loci as a single band, so a third primer (F2) was designed for nested PCR using the same thermocycler conditions for greater specificity. The number of parsimony informative characters ranged from 20 to 144 , and the amount of pairwise variation ranged from a minimum of $0.007-0.055$ to a maximum of $0.055-0.281$. $d 8$ and rep 1 provide the most informative characters, with pairwise variation from $0.031-0.209$ between sequences.

\section{CONCLUSIONS}

Combining genetic and genomic resources within the grasses is a powerful approach for designing phylogenetic markers. The 11 loci targeted for primer design in this study belong to a diverse set of genes that are known to influence plant and inflorescence architecture. These loci are distributed on nine of the 10 maize chromosomes, suggesting they are unlinked and providing independent records of duplications and gene loss within the tribe. Cloning PCR products and sequencing multiple clones allows the identification of paralogues, helping to identify allopolyploid events and reducing conflict between individual phylogenetic trees. These markers offer a valuable alternative to the nuclear-encoded ITS locus.

\section{LITERATURE CITED}

Álvarez, I., AND J. F. Wendel. 2003. Ribosomal ITS sequences and plant phylogenetic inference. Molecular Phylogenetics and Evolution 29: 417-434.

Bortiri, E., G. Chuck, E. Vollbrecht, T. Rocheford, R. MartiensSen, AND S. HAKE. 2006. ramosa2 encodes a LATERAL ORGAN
BOUNDARY domain protein that determines the fate of stem cells in branch meristems of maize. Plant Cell 18: 574-585.

Gadberry, M., S. Malcomber, A. Doust, and E. Kellogg. 2005. Primaclade-A flexible tool to find conserved PCR primers across multiple species. Bioinformatics (Oxford, England) 21: 1263-1264.

Gallavotti, A., J. A. Long, S. Stanfield, X. Yang, D. Jackson, E. Vollbrecht, AND R. J. Schmidt. 2010. The control of axillary meristem fate in the maize ramosa pathway. Development 137: 2849-2856.

Grass Phylogeny Working Group. 2001. Phylogeny and subfamilial classification of the Poaceae. Annals of the Missouri Botanical Garden 88: 373-457.

Ikeda, K., N. Nagasawa, and Y. Nagato. 2005. ABERRANT PANICLE ORGANIZATION 1 temporally regulates meristem identity in rice. Developmental Biology 282: 349-360.

Jang, S., B. Lee, C. Kim, S.-J. Kim, J. Yim, J.-J. Han, S. Lee, et AL. 2003. The OsFORI gene encodes a polygalacturonase-inhibiting protein (PGIP) that regulates floral organ number in rice. Plant Molecular Biology 53: 357-372.

Li, X., Q. Qian, Z. Fu, Y. Wang, G. Xiong, D. Zeng, X. Wang, et al. 2003. Control of tillering in rice. Nature 422: 618-621.

Moreno, M., L. Harper, R. Krueger, S. Dellaporta, and M. Freeling. 1997. liguleless 1 encodes a nuclear-localized protein required for induction of ligules and auricles during maize leaf organogenesis. Genes \& Development 11: 616-628.

Peng, J., D. E. Richards, N. M. Hartley, G. P. Murphy, K. M. Devos, J. E. Flintham, J. Beales, et AL. 1999. 'Green revolution' genes encode mutant gibberellin response modulators. Nature 400: 256-261.

Phillips, K. A., A. L. Skirpan, X. Liu, A. Christensen, T. L. Slewinski, C. Hudson, S. BARAZESH, ET AL. 2011. vanishing tassel2 encodes a grass-specific tryptophan aminotransferase required for vegetative and reproductive development in maize. Plant Cell 23: 550-566.

Punta, M., P. C. Coggill, R. Y. Eberhardt, J. Mistry, J. Tate, C. Boursnell, N. Pang, et al. 2012. The Pfam protein families database. Nucleic Acids Research 40(D1): D290-D301.

Swofford, D. 2002. PAUP 4.0: Phylogenetic analysis using parsimony and other methods. Sinauer, Sunderland, Massachusetts, USA.

Vollbrecht, E., P. S. Springer, L. Goh, E. S. Buckler Iv, and R. MartiEnssen. 2005. Architecture of floral branch systems in maize and related grasses. Nature 436: 1119-1126.

Yuan, Z., S. GaO, D.-W. Xue, D. Luo, L.-T. Li, S.-Y. Ding, X. Yao, ET AL. 2009. RETARDED PALEA1 controls palea development and floral zygomorphy in rice. Plant Physiology 149: 235-244.

Zhu, K., D. Tang, C. Yan, Z. Chi, H. Yu, J. Chen, J. Liang, et al. 2010. Erect panicle 2 encodes a novel protein that regulates panicle erectness in Indica rice. Genetics 184: 343-350. 
APPENDIX 1. List of taxa sampled with voucher information and GenBank accession numbers.

\begin{tabular}{|c|c|c|c|c|c|c|c|c|c|c|c|c|c|c|}
\hline \multirow[b]{2}{*}{ Taxa sampled } & \multirow{2}{*}{$\begin{array}{l}\text { Voucher } \\
\text { (Herbarium) }\end{array}$} & \multicolumn{13}{|c|}{ GenBank accession no. } \\
\hline & & apo1 & $d 8$ & $e p 2 \_e x 7$ & ep2_ex8 & for 1 & $\lg 1$ & moc1_A & moc1_B & $r a 1$ & $r a 2$ & rel2 & rep 1 & $v t 2$ \\
\hline $\begin{array}{l}\text { Andropogon hallii } \\
\text { Hack. }\end{array}$ & Kellogg PI477973 (A) & JQ951748 & JQ951713 & JQ951705 & JQ951694 & JQ951684 & JQ951738 & JQ951670 & JQ951662 & JQ951653 & JQ951644 & - & JQ951634 & JQ951731 \\
\hline $\begin{array}{l}\text { Andropterum } \\
\text { stolzii (Pilg.) C. } \\
\text { E. Hubb. }\end{array}$ & $\begin{array}{l}\mathrm{SM} 3091,(\mathrm{MO})^{\circ} \\
09^{\circ} 13^{\prime} \mathrm{S}, 034^{\circ} 56^{\prime} \mathrm{E}\end{array}$ & JQ951745 & JQ951707 & JQ951698 & JQ951690 & JQ951681 & JQ951734 & JQ951668 & JQ951663 & JQ951651 & JQ951643 & JQ951718 & JQ951631 & JQ951725 \\
\hline $\begin{array}{l}\text { Chrysopogon } \\
\text { gryllus (L.) Trin. }\end{array}$ & Kellogg PI250984 (A) & JQ951750 & JQ951709 & JQ951699 & JQ951687 & JQ951680 & JQ951739 & JQ951674 & JQ951667 & JQ951655 & JQ951640 & JQ951717 & JQ951636 & - \\
\hline $\begin{array}{l}\text { Coelorachis } \\
\text { lepidura Staph. }\end{array}$ & $\begin{array}{l}\mathrm{SM} 3060,(\mathrm{MO}) \\
06^{\circ} 28^{\prime} \mathrm{S}, 038^{\circ} 49^{\prime} \mathrm{W}\end{array}$ & JQ951743 & JQ951708 & JQ951697 & JQ951692 & JQ951677 & JQ951740 & JQ951671 & JQ951661 & JQ951649 & - & - & JQ951635 & JQ951726 \\
\hline $\begin{array}{l}\text { Cymbopogon } \\
\text { flexuosus (Nees } \\
\text { ex Steud.) Stapf }\end{array}$ & Kellogg PI209700 (A) & JQ951744 & JQ951710 & JQ951700 & JQ951689 & JQ951679 & JQ951733 & JQ951669 & JQ951666 & JQ951650 & JQ951641 & JQ951722 & JQ951632 & JQ951730 \\
\hline $\begin{array}{l}\text { Dichanthium } \\
\text { annulatum } \\
\text { (Forssk.) Stapf }\end{array}$ & Kellogg PI240155 (A) & JQ951749 & JQ951711 & JQ951701 & JQ951688 & JQ951682 & JQ951735 & JQ951672 & JQ951658 & JQ951656 & JQ951642 & JQ951719 & JQ951630 & JQ951728 \\
\hline $\begin{array}{l}\text { Loudetia Hochst. } \\
\text { ex Steud. }\end{array}$ & $\begin{array}{l}\mathrm{SM} 3084,(\mathrm{MO}) \\
08^{\circ} 11^{\prime} \mathrm{S}, 034^{\circ} 54^{\prime} \mathrm{E}\end{array}$ & JQ951742 & JQ951706 & JQ951696 & JQ951686 & JQ951676 & JQ951732 & - & JQ951659 & JQ951648 & JQ951639 & JQ951720 & JQ951629 & JQ951724 \\
\hline $\begin{array}{l}\text { Phacelurus } \\
\text { digitatus (Sibth. } \\
\text { \& Sm.) Griseb. }\end{array}$ & Kellogg PI206746 (A) & JQ951747 & JQ951714 & JQ951702 & JQ951691 & JQ951678 & JQ951736 & - & JQ951665 & JQ951652 & JQ951646 & JQ951716 & JQ951637 & JQ951727 \\
\hline $\begin{array}{l}\text { Schizachyrium } \\
\text { hirtiflorum Nees }\end{array}$ & Kellogg PI216107 (A) & JQ951746 & JQ951715 & JQ951704 & JQ951695 & JQ951685 & JQ951737 & JQ951673 & JQ951660 & JQ951654 & JQ951645 & JQ951721 & JQ951633 & - \\
\hline $\begin{array}{l}\text { Sorghum bicolor } \\
\text { (L.) Moench }\end{array}$ & Kellogg PI255738 (A) & JQ951751 & JQ951712 & JQ951703 & JQ951693 & JQ951683 & JQ951741 & JQ951675 & JQ951664 & JQ951657 & JQ951647 & JQ951723 & JQ951638 & JQ951729 \\
\hline
\end{tabular}

